\title{
Fertilização In Vitro com Ciclos Programados de Baixo Custo - Avaliação de Resultados Iniciais de um Centro de Reprodução Humana de Hospital de Ensino
}

In vitro Fertilization with Low-cost Programmed Cycles - First Outcome in a Teaching Hospital

Nelson Antunes Júnior, Georgia Haik Badra, Emerson Barchi Cordts, Waldemar Almeida Pereira de Carvalho, Phillip Wolff, Caio Parente Barbosa, Sérgio Peixoto

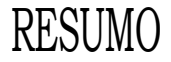

Objetivos: avaliação dos resultados iniciais de fertilização in vitro (FIV) em instituição universitária, empregando ciclos programados de baixo custo.

Métodos: entre maio e dezembro de 2002, foram iniciados 66 ciclos programados de FIV, utilizando acetato de noretisterona, citrato de clomifeno e gonadotrofina coriônica humana (hCG). A punção folicular guiada por ultra-sonografia foi realizada 34 a 36 horas após a administração de hCG e a transferência, 48 horas após a punção. A gestação foi considerada clínica após visualização de batimentos cardíacos à ultra-sonografia transvaginal.

Resultados: a taxa de cancelamento foi de 21,2\%. Em média, 2,8 folículos e 1,7 oócitos foram obtidos por punção. Em 79,6\% dos ciclos puncionados recuperaram-se oócitos, que foram fertilizados em 69\% dos casos. O número de embrióes por transferência foi de 1,5. Houve algum grau de dificuldade em 10,2\% das punções e 32,4\% das transferências realizadas. A taxa de gestação obtida foi de $10,8 \%$ por transferência, entretanto, o custo com medicação por embrião transferido foi de apenas $R \$ 96,00$.

Conclusão: evidenciam-se as dificuldades de iniciar um programa de FIV em instituição de ensino, sem fins lucrativos e voltada à população carente. Com a prática, a taxa cumulativa de gravidez tende a ser semelhante às dos centros de referência, porém com custo e incidência de complicações significativamente inferiores.

PALAVRAS-CHAVE: Fertilização in vitro.Reprodução assistida. Clomifeno. Baixo custo.

\section{Introdução}

Em 2003, Louise Brown completou 25 anos de vida. O seu nascimento, em julho de 1978, num pequeno hospital situado no norte da Inglaterra, foi resultado da primeira tentativa bem sucedida de fertilização in vitro (FIV), fusão do conhecimento do ginecologista Patrick Steptoe, pioneiro em laparoscopia, e do cientista Robert Edwards e re-

Setor de Reprodução Humana - Disciplina de Ginecologia e Obstetricia da Faculdade de Medicina do ABC - Santo André - SP

Correspondência:

Nelson Antunes Jr

Rua Almirante Pereira Guimarães, 360 - Pacaembu

01250-000 - São Paulo - SP

Tel/Fax: (11) 3871-2466

e-mail: ghbadra@uol.com.br presentou, sem dúvida, um dos principais marcos na história da Reprodução Humana ${ }^{1}$.

Desde então, em todo o mundo, aproximadamente 1 milhão de bebês nasceram como fruto da tecnologia da reprodução assistida.

Com o sucessivo aprimoramento técnico e descoberta de novas drogas para hiperestimulação deliberada dos ovários, têm aumentado progressivamente as chances de gravidez em casais inicialmente considerados inférteis. Por outro lado, aumentaram também os riscos de gestações múltiplas e o custo para concretizar esse sonho, inclusive nos escassos serviços públicos existentes ${ }^{2}$.

A incidência de infertilidade nos países em desenvolvimento em que predomina a população de baixa renda, como é o caso do Brasil, é elevada. Além da alta incidência de doenças inflamatórias 
pélvicas e laqueadura tubária sem possibilidade de reanastomose, os homens menos favorecidos economicamente têm mais doenças sexualmente transmissiveis e são os mais expostos ocupacionalmente aos poluentes, substâncias químicas e fatores físicos que modificam as características espermáticas ${ }^{2,3}$.

Infelizmente, a maioria da população brasileira não tem acesso a serviços de reprodução humana ou condições financeiras para arcar com os altos custos de medicação e procedimentos em reprodução assistida ${ }^{4}$. Por muitos anos, restou a estes casais menos favorecidos aguardar em intermináveis filas de espera ou simplesmente aceitar sua condição e decidir-se pela adoção.

Com o intuito de suprir a necessidade da população mais carente da região, foi inaugurado, em janeiro de 2002, o Centro de Reprodução Humana da Faculdade de Medicina do ABC.

Por meio da indução da ovulação com drogas de preço mais acessível e pagamento apenas do custo dos materiais descartáveis e meio de cultura, foi possivel iniciar, no primeiro ano de funcionamento, 66 ciclos de FIV, além de uma série de procedimentos menos complexos, sem receber qualquer incentivo econômico.

O presente estudo tem como objetivo apresentar os resultados iniciais de FIV do Centro de Reprodução Humana, procurando identificar, de forma crítica, as características que levaram a tais resultados e as dificuldades de se iniciar um programa deste tipo em instituição de ensino, sem fins lucrativos e com recursos financeiros limitados, propondo, ainda, algumas possibilidades para o futuro.

\section{Pacientes e Métodos}

No período entre maio e dezembro de 2002 foram iniciados, em 45 pacientes, 66 ciclos programados de FIV no Centro de Reprodução Humana. O levantamento retrospectivo destes casos foi aprovado pela Comissão de Ética em Pesquisa da Faculdade de Medicina do ABC.

Todas as pacientes selecionadas para o presente estudo estavam em acompanhamento no ambulatório de Reprodução Humana da Disciplina de Ginecologia e Obstetrícia e foram inicialmente submetidas a pesquisa básica de infertilidade conforme o protocolo utilizado no serviço. Foram realizados os seguintes exames de triagem: hemograma, tipagem sanguinea, glicemia de jejum, dosagens hormonais - FSH, LH, estradiol ( $3^{\circ}$ dia do ciclo menstrual), TSH, $\mathrm{T}_{4}$ livre, prolactina, progesterona $\left(21^{\circ}\right.$ dia do ciclo),sorologias para HIV, hepatite $\mathrm{B}$, hepatite $\mathrm{C}$, rubéola, toxoplasmose e citomegalovirus, ultrasonografia (USG) transvaginal, histerossalpingografia, histeroscopia e espermograma do parceiro. Em algumas situações específicas, associouse também a dosagem de androgênios. Todos os casos com fator masculino presente foram concomitantemente acompanhados pela equipe de andrologia da instituição.

Foi realizado pelo menos um espermograma com processamento seminal por migração ascendente (swim-up) antes da inclusão do casal no grupo para FIV.

Foram excluídos todos os casais com fator masculino acentuado, caracterizado pela concentração de espermatozóides após processamento inferior a 3 milhões $/ \mathrm{mL}$, e aqueles nos quais identificaram-se sinais de falência ovariana (FSH $>15,0$ ) ou anormalidades anatômicas passiveis de correção cirúrgica.

As 45 pacientes submetidas a FIV tinham idades entre 24 e 45 anos, em média, 34,5. Devese frisar que quase um terço $(31,1 \%)$ tinha mais de 37 anos de idade. As idades dos parceiros variavam de 22 a 56 anos, em média, 39 anos.

Em todos os casos, foi identificado algum fator feminino de infertilidade. A infertilidade primária esteve presente em $40 \%$ do total. Em mais de $93 \%$ das mulheres, o fator identificado foi tuboperitoneal. Deve-se frisar que $31,1 \%$ das pacientes eram laqueadas e sem possibilidade de reanastomose tubárea após avaliação laparoscópica. Apenas $22,2 \%$ dos casais apresentavam algum fator masculino associado, que não contraindicava a realização de FIV.

A laparoscopia diagnóstica foi anteriormente realizada em $82,2 \%$ de todas as pacientes. Apenas $8(17,7 \%)$ não foram submetidas ao procedimento, por recusar a realização de cirurgia.

Das 45 pacientes, $64,4 \%$ nunca foram submetidas a nenhum tratamento específico para engravidar, exceto a cirurgia citada. Dentre as demais, $6,6 \%$ já haviam sido submetidas a ciclos de coito programado sem sucesso; $11,1 \%$ tinham tentativas anteriores frustradas de inseminação intrauterina, $11,1 \%$ tinham tentado FIV em outros serviços e $6,6 \%$ já haviam sido submetidas a mais de um tipo de procedimento em reprodução assistida.

Antes do início do ciclo de indução, todos os casais foram cuidadosamente orientados, assinaram o termo de consentimento específico para FIV e passaram por avaliação psicológica. Além disso, todas as pacientes receberam amostras gratuitas de $1 \mathrm{~g}$ de azitromicina que utilizaram profilaticamente, por via oral. 
Com o objetivo de programar os ciclos, tornando-os sincronizados, as pacientes foram bloqueadas na segunda fase do ciclo menstrual anterior com acetato de noretisterona, $10 \mathrm{mg}$ via oral ao dia, por um período variável de 9 a 66 dias.

Foi considerado o dia zero $\left(D_{0}\right)$ do ciclo o primeiro dia em que não se utilizou a droga citada anteriormente. A ovulação foi induzida com citrato de clomifeno, $150 \mathrm{mg}$ via oral ao dia por 5 dias consecutivos, iniciando-se no $7^{\circ}$ dia do ciclo $\left(D_{7}\right)$.

A resposta à indução foi controlada por USG transvaginal realizada no $15^{\circ}$ dia do ciclo $\left(D_{15}\right)$. Se, neste dia, nenhum folículo tivesse mais do que $14 \mathrm{~mm}$ de diâmetro médio, considerava-se falha de indução e havia cancelamento do ciclo. Para as pacientes que tiveram resposta folicular adequada, neste dia foi administrada gonadotrofina coriônica humana (hCG) - 5000 UI intramuscular, independentemente do diâmetro folicular.

A punção folicular guiada por USG foi realizada em centro cirúrgico, sob sedação com fentanil e propofol, 34 a 36 horas após a administração de hCG.

Cada oócito identificado no fluido folicular foi lavado em meio de cultura (fluido tubárico humano modificado, transferido para a cavidade central da placa contendo meio de inseminação (fluido tubárico humano e substituto sintético do soro, a $7,5 \%$ ) e colocado na estufa a $37^{\circ} \mathrm{C}$ em atmosfera de $5 \%$ de $\mathrm{CO}_{2}$ em ar. Até 6 horas após o início da incubação dos oócitos, cada um deles foi exposto a cerca de 100.000 espermatozóides móveis processados do parceiro.

Os embriões formados foram transferidos para o interior da cavidade endometrial através do colo uterino, cerca de 48 horas após a punção, utilizando-se catéter flexível Tom Cat. Após a transferência, as pacientes permaneceram em repouso absoluto por aproximadamente 30 minutos e foram orientadas a colher sangue para dosagem de $\beta$-hCG quantitativo após 14 dias.

A gestação foi clinicamente diagnosticada pela visualização de batimentos cardíacos na USG transvaginal.

As gestantes foram encaminhadas para o setor de Pré-Natal de Alto Risco da Disciplina de Ginecologia e Obstetrícia da instituição para acompanhamento.

\section{Resultados}

Dos 66 ciclos programados iniciados, 14 foram suspensos antes que fosse realizada a punção folicular, sendo 10 deles por ausência de res- posta adequada à indução e quatro devido a sinais de luteinização precoce após administração de hCG. A taxa de cancelamento foi de $21,2 \%$.

Nos 52 ciclos restantes, a resposta obtida correspondeu às expectativas, entretanto em três desses casos $(5,7 \%)$ não se realizou a punção folicular por problemas técnicos. Foi, portanto, realizada a punção em 49 dos ciclos iniciados. No total, foram aspirados 141 folículos de diâmetro maior ou igual a $15 \mathrm{~mm}$, o que representou, em média, 2,87 folículos por punção.

Em 10,2\% dos casos houve algum grau de dificuldade para realizar a punção, como presença de sangue ou coágulos no líquido aspirado, extravasamento de fluido folicular durante o procedimento, falhas no sistema de aspiração ou dificuldade de acesso aos ovários.

Não foram observadas complicações relacionadas à indução da ovulação ou aos procedimentos anestésico e cirúrgico.

Nos 141 folículos puncionados foram identificados 84 oócitos (59,5\%). A média de oócitos obtidos por punção foi de 1,7. Em 39 dos ciclos com punção $(79,6 \%)$ recuperou-se pelo menos um oócito e em $37(94,9 \%)$ destes casos houve fertilização. A taxa de fertilização foi de $69 \%$ e os 58 embriões formados foram todos transferidos.

Em média, o número de embriões por transferência foi de 1,56. Em apenas três ciclos $(8,1 \%)$ foram transferidos mais de dois embriões.

Em $32,4 \%$, ou seja, em quase um terço das 37 transferências realizadas houve algum tipo de dificuldade, como contaminação do cateter com sangue ou muco, necessidade de dilatação do canal cervical ou pinçamento do colo uterino com pinça de Pozzi, além de retenção embrionária no cateter e necessidade de nova tentativa.

O custo de medicação por embrião transferido no presente estudo foi de aproximadamente $\mathrm{R} \$ 96,00$.

O número de ciclos com gestação clinicamente comprovada pela presença de batimentos cardíacos à USG transvaginal foi de quatro, todas em pacientes com até 30 anos de idade. Não houve nenhum caso de abortamento, gestação ectópica ou gestação múltipla. Não foram considerados os casos de gestação bioquímica. A taxa de gestação por ciclo iniciado foi de 6,3 e 9,5\% por paciente, excetuando-se os três casos suspensos por problemas técnicos antes da punção. A taxa de gestação por transferência realizada foi de $10,8 \%$.

Cerca de $62 \%$ dos casais foram submetidos a apenas uma tentativa de FIV, 29\% a duas e 9\% a três tentativas de FIV com o mesmo protocolo. Das quatro gestações obtidas, duas ocorreram na primeira tentativa, uma na segunda e a outra no terceiro ciclo programado de FIV. 
As quatro gestações evoluíram bem e sem intercorrências obstétricas ou clínicas significativas. Em nenhum dos casos houve necessidade de internação durante o pré-natal. Todos os bebês nasceram a termo, com pesos considerados adequados para a idade gestacional e obtiveram alta hospitalar juntamente com as respectivas mães.

\section{Discussão}

Em 1978, Lopata et al. ${ }^{5}$ foram os primeiros a usar o citrato de clomifeno associado a hCG em programas de FIV e transferência embrionária. A primeira gestação obtida com este tipo de estimulação foi relatada por Trounson et al. ${ }^{6}$, em 1981.

Com o uso dos ciclos hiperestimulados, houve significativo acréscimo nas taxas de gestação, porém a necessidade de exaustiva monitorização folicular e a total falta de previsão do dia oportuno para a captação dos ovócitos ainda eram fatores que dificultavam o controle e aumentavam o custo operacional do procedimento ${ }^{7}$.

Com o objetivo de simplificar os esquemas utilizados, visando diminuir os custos e a freqüência de monitorização, além de definir com antecedência as datas de captação ovular, alguns autores passaram a utilizar os chamados ciclos programa$\operatorname{dos}^{8-10}$. O sincronismo das pacientes em programação de FIV era obtido pelo prolongamento ou encurtamento do ciclo menstrual anterior pela administração, por periodos variáveis, de contraceptivos orais combinados ou progestagênios. Em seguida, iniciava-se esquema fixo de hiperestímulo ovariano ${ }^{7}$.

O ciclo programado descrito por Templeton et al. ${ }^{8}$, em 1984, nos pareceu o mais adequado para iniciar um programa de FIV em instituição de ensino, por apresentar menor custo financeiro do que aquele proposto em 1986, por Frydman et al. ${ }^{10}$, além de não apresentar, teoricamente, nenhum efeito colateral significativo para as pacientes.

Ambos os autores citados anteriormente referem ter tido resultados discretamente melhores nas pacientes bloqueadas com o progestagênio do que naquelas que utilizaram contraceptivo oral combinado, o que justificou, neste estudo, a escolha pela utilização da noretisterona para realizar o bloqueio dos ciclos e sincronizar as pacientes.

A publicação isolada de bons resultados por serviços de reprodução humana não tem, a rigor, valor científico, pois existem diversas variáveis envolvidas que podem levar a uma interpretação inadequada, além da possibilidade de manipulação dos dados.
Uma forma de manipulação muito freqüentemente utilizada é a exclusão dos resultados de pacientes com fatores de mau prognóstico para engravidar $^{11}$, como, por exemplo, a idade avançada. Deve-se frisar que alguns serviços públicos sequer matriculam a paciente acima de uma certa idade ${ }^{12}$, mesmo que sua função ovariana esteja preservada.

A idade da mulher é considerada o fator mais importante na determinação dos resultados de FIV. Após os 36 anos de idade, já há significativa queda nas taxas de gestação e nascidos vivos, que pioram significativamente após os $40 \operatorname{anos}^{13}$.

No presente estudo, aproximadamente um terço das pacientes submetidas a FIV tinha idade igual ou superior a 38 anos e, destas, quase $43 \%$ já ultrapassavam os 40 . Se tivéssemos excluído aquelas com mais de 37 anos, a taxa de gestação por transferência seria de $13,8 \%$, uma vez que todas as pacientes que engravidaram tinham no máximo 30 anos de idade.

Outro fator que deve ser levado em consideração é o fato de $22,2 \%$ dos casais deste grupo apresentarem algum fator masculino, discreto ou moderado, associado ao fator feminino. Estudos recentes têm demonstrado que existe redução de aproximadamente $10 \%$ das taxas de sucesso em casos de infertilidade masculina tratada por meio da FIV convencional ${ }^{14}$. Como a micromanipulação de gametas ainda não está disponível no serviço, a indicação de ICSI acaba sendo mais restrita do que nos centros que já contam com o procedimento e, conseqüentemente, há maior maleabilidade nas indicações de FIV, o que talvez possa ter interferido nos resultados, embora a nossa taxa de fertilização tenha sido satisfatória. Em breve, com a aquisição do micromanipulador de gametas, será possível pesquisar se existe ou não tal interferência.

A taxa de cancelamento que obtivemos foi de $21,2 \%$, ou seja, em 14 dos 66 ciclos iniciados não foi realizada punção. Deve-se frisar que, em quatro destes casos, houve resposta folicular satisfatória à indução, porém no dia da punção verificaram-se sinais de luteinização à USG após utilização de hCG, e conseqüentemente, o procedimento foi suspenso. Ao questionar as pacientes sobre o rigor do horário em que receberam a medicação, não foi possivel excluir a possibilidade de erro por motivos sociais como, por exemplo, aproveitar alguém disponivel para aplicar a injeção gratuitamente. Além disso, em cerca de $10 \%$ dos casos, a punção folicular demorou mais do que era previsto, devido às dificuldades já citadas e à curva de aprendizagem dos residentes. Se levarmos em consideração tal tipo de interferência humana, a taxa de cancelamento cai para $15,1 \%$. 
Templeton et al. ${ }^{8}$ e Braude et al. ${ }^{9}$, em 1984, Messinis et al. ${ }^{15}$, em 1986, e Tognotti et al. ${ }^{7}$, em 1989 , realizaram ciclos programados com protocolos de indução semelhantes ao deste estudo, com objetivo de pesquisa, em pacientes submetidas a laparoscopia (Tabela 1).
Pela análise da Tabela 1, pode-se verificar que os nossos resultados foram um pouco superiores àqueles descritos na literatura. Provavelmente, essa pequena diferença deva-se ao fato de termos realizado as punções guiadas por USG, enquanto os demais autores citados utilizaram a laparoscopia.

Tabela 1 - Ciclos programados com CC + hCG - Dados comparativos da literatura

\begin{tabular}{|c|c|c|c|c|c|}
\hline & Templeton et al..$^{8}$ & Braude et al. ${ }^{9}$ & Messinis et al. ${ }^{15}$ & Tognotti et al. ${ }^{7}$ & Estudo atual \\
\hline Média folículos/punção & * & * & $1,8(a)$ & $2,3(b)$ & $2,8(b)$ \\
\hline \% de folículos comrecuperação de ovócitos & * & * & $81 \%$ & $52,2 \%$ & $59,5 \%$ \\
\hline Média ovócitos/punção & * & 1,3 & 1,5 & 1,2 & 1,7 \\
\hline$\%$ de ciclos puncionados com pelo menos 1 ovócito & $63 \%$ & $76 \%$ & * & $70 \%$ & $79,6 \%$ \\
\hline
\end{tabular}

Modificado de Tognotti et al. (1989)7.

$\mathrm{CC}$ - citrato de clomifeno

*Não há dados relatados.

(a) - Diâmetro folicular médio $>16 \mathrm{~mm}$.

(b) - Diâmetro folicular médio $\geq 15 \mathrm{~mm}$.

No presente estudo, em cerca de $20 \%$ dos ciclos puncionados não foram recuperados oócitos. Alguns autores relacionam a ausência de recuperação de oócitos após aspiração folicular a atresia oocitária precoce e à anormalidade da atividade biológica de lotes de hCG comercializada ${ }^{16}$. Quintans et al. ${ }^{17}$, em 1998, sugerem que grande parte dos casos esteja associada a erro humano com relação ao horário de administração da ampola de hCG, que tem como função finalizar a maturação folicular. Referem, ainda, que uma segunda dose de hCG seguida de nova punção permite a recuperação satisfatória de oócitos em grande parte das pacientes ${ }^{16,17}$.

Curiosamente, neste estudo, em todos os casos em que houve dificuldade de punção, não se identificou nenhum oócito no líquido aspirado, o que representa cerca de metade dos ciclos sem recuperação de oócitos. Além disso, chama a atenção o fato de que aproximadamente metade dos ciclos sem oócitos identificados foram realizados em mulheres com mais de 37 anos de idade.

No grupo de pacientes em que a transferência embrionária foi considerada fácil $(67,5 \%)$, a taxa de gestação clínica obtida foi de $16 \%$. No grupo em que houve algum tipo de dificuldade $(32,4 \%)$ nenhuma paciente engravidou. Embora não haja consenso, acredita-se que a dificuldade de introdução do cateter de transferência através do orifício interno do colo pode causar um efeito devastador sobre o resultado do ciclo ${ }^{18}$.

Por tratar-se de uma instituição de ensino, os procedimentos são, na maioria das vezes, executados por residentes em treinamento, sob supervisão direta de membros da equipe com mais experiência. Neste sentido, algumas falhas acabam sendo inevitáveis e fazem parte da curva de aprendizado; infelizmente, também acabam por comprometer os resultados finais.

Esta relação entre experiência da equipe e sucesso da FIV é fortemente sustentada por dados de literatura; verifica-se associação direta entre o número de ciclos realizados por ano e as taxas de gravidez encontradas nos diferentes serviços ${ }^{4}$.

Com o passar dos anos, visando aumentar as taxas de gestação, os protocolos de estimulação ovariana foram aumentando progressivamente a quantidade e tipos de drogas utilizadas. O uso isolado do citrato de clomifeno em FIV foi praticamente abandonado, em virtude dos resultados menos significativos quando comparado aos demais protocolos.

Com a utilização dos análogos de GnRH, por exemplo, houve significativa redução da taxa de cancelamento dos ciclos, por meio da supressão quase total dos casos de luteinização precoce e, conseqüentemente, aumento das taxas de gestação. Por outro lado, a síndrome de hiperestímulo ovariano, a freqüente intolerância aos efeitos colaterais como dores de cabeça, queda de cabelos, ganho de peso, diminuição da libido, dor abdominal e, principalmente, a alta incidência de gestações múltiplas e todas as suas conseqüências tornaram-se mais freqüentes com o uso deste tipo de medicação ${ }^{19}$.

Uma prática que se tornou muito comum ao longo dos anos, nos centros de reprodução humana, foi aumentar o número de embriões transferidos por ciclo, na tentativa de melhorar as taxas 
de sucesso ${ }^{14}$; com isso, também aumentaram significativamente as taxas de gestações múltiplas de diversas ordens. Recentemente, sucessivos estudos têm demonstrado que a qualidade dos embriões e do endométrio parece ser fator mais importante do que o número de embriões propriamente dito ${ }^{20}$.

Indiscutivelmente, quando existe um maior número de embriões formados, é possível escolher os melhores para transferir e, com isso, aumentam as taxas de implantação e gestação ${ }^{13}$. Entretanto, essa seleção só é possivel em serviços que tenham disponível a criopreservação de embriões, uma vez que o descarte em nosso meio é proibido. Em virtude do número reduzido de embriões por transferência (em média 1,5$)$, não houve seleção da qualidade dos embriões no presente estudo, já que todos os embriões formados foram transferidos. Também não ocorreu nenhum caso de gestação múltipla.

Não se pode considerar a gestação múltipla como procedimento bem sucedido. Em comparação com uma gestação única, a chance de ter uma criança saudável em casa é significativamente menor nestes casos ${ }^{19}$. Além de todas as complicações obstétricas e neonatais, a mortalidade perinatal é cerca de 5 a 6 vezes maior ${ }^{21}$. Além disso, devem-se também considerar as complicações emocionais e financeiras a longo prazo.

Atualmente, discute-se em todo o mundo a necessidade de as estatísticas em reprodução assistida passarem a incluir também as taxas de gestação múltipla, redução fetal, morbimortalidade materna e perinatal e conseqüências dos procedimentos realizados ${ }^{22}$. Alguns sugerem que seja instituída a taxa de "nascimento por embrião transferido", que refletiria a chance de ter um bebê saudável em casa e, portanto, as reais taxas de sucesso de cada centro de reprodução humana ${ }^{21}$.

A Federação Internacional de Ginecologia e Obstetrícia (FIGO) recomenda que o objetivo da reprodução assistida deve ser sempre a gestação única e refere-se ao abuso de medicações para indução de ovulação e transferência de elevado número de embriões como iatrogenia ${ }^{22}$. Sugere, ainda, que a possibilidade de redução fetal deve ser considerada uma alternativa em casos de gestação tripla ou superior, em virtude dos elevados riscos materno-fetais implicados ${ }^{22}$.

Mesmo nos países em que a redução embrionária é permitida, o que não é o caso do Brasil, há uma série de implicações éticas e psicológicas ${ }^{23}$, além dos potenciais riscos do procedimento sobre os resultados da própria gestação ${ }^{24}$, que não devem ser ignorados.
Talvez, como sugerem Olivennes e Frydman ${ }^{19}$, em 1998, devamos tentar resgatar o objetivo inicial de Patrick Steptoe e Robert Edwards - produzir in vitro um embrião que possa levar ao nascimento de uma criança saudável ${ }^{1}$. Talvez esteja na hora de repensar se realmente vale a pena ser tão agressivo em reprodução humana; os mesmos autores sugerem que a FIV seja mais "amigável", de menor custo físico, emocional, social e financeiro. Para tanto, além da diminuição do número de embriões transferidos e estímulo às pesquisas voltadas à implantação embrionária, sugerem que os protocolos de indução sejam simplificados de diversas maneiras, entre elas, com o retorno, inclusive, do uso do citrato de clomifeno.

Estima-se que apenas 0,7 a 2,8\% das mulheres que poderiam se beneficiar com algum tipo de tratamento para infertilidade realmente tenham acesso a ele ${ }^{25}$. Aproximadamente $90 \%$ dos centros de reprodução humana da América Latina são instituições particulares ${ }^{25}$ e cobram, por tentativa, valores que variam de US\$ 3000,00 a US\$ $11000,00^{4}$. De acordo com publicação da Organização Mundial de Saúde (OMS) em 2002, em uma instituição pública do interior de São Paulo, por exemplo, cada casal paga entre US\$ 750,00 e US\$ 2000,00 por ciclo sem criopreservação de gametas ou embriões ${ }^{2}$. Em valores atuais, o custo com medicação para realizar um ciclo de FIV conforme o protocolo de um conceituado serviço público de São Paulo em que se utiliza anticoncepcional oral, análogo de GnRH, FSH recombinante e hCG é de aproximadamente $\mathrm{R} \$ 3700,00$ (Tabela 2).

Uma vez que a medicação deve ser custeada pelo casal, o nosso tipo de população-alvo justifica, por si só, o tipo de protocolo escolhido, no qual o custo total com medicação é de, no máximo, $\mathrm{R} \$ 150,00$, ou seja, cerca de $4 \%$ do valor citado anteriormente. No presente estudo, o custo com medicação foi de $\mathrm{R} \$ 96,00$ por embrião transferido. Deste modo, podemos oferecer à população de baixa renda a chance de tentar três vezes utilizando protocolo mais simples e barato com uma taxa de cerca de $10 \%$ de sucesso por tentativa, ao invés de se submeter a uma única tentativa cuja taxa de gestação é de cerca de $30 \%$ com elevada incidência de gestação múltipla, efeitos colaterais muitas vezes desagradáveis e dificuldades financeiras a perder de vista ${ }^{19}$.

Considerando-se que a tendência mundial é a redução do número de embriões transferidos por ciclo para um ou dois, no máximo, a relação custo-benefício não justifica a realização de qualquer tipo de protocolo de indução mais elaborado em um serviço sem condições imediatas de criopreservação. Não tem sentido um casal que recebe salário mínimo gastar cerca de 25 vezes a 
Tabela 2 - Relação custo-benefício de dois diferentes esquemas de indução para FIV*.

\section{Esquema de indução}

Acetato de noretisterona

Citrato de clomifeno

hCG

Anticoncepcional oral

Análogo de $\mathrm{GnRH}$

FSH recombinante

hCG

*Considerando a quantidade média de medicação utilizada por ciclo em cada protocolo.

**Fonte: Lista de preços da Revista Guia da Farmácia n 122 / janeiro 2003.

mais para ter um maior número de embriões formados, se estes embriões não deverão ser transferidos e também não poderão ser congelados.

Em todo programa inicial existe um período de adaptação, no qual são computados os acertos, os erros e sugeridas as mudanças consideradas necessárias. De acordo com Gabiatti et al. ${ }^{4}$, o programa de FIV da UNICAMP iniciado em 1991 só obteve a primeira gestação após 2 anos de tentativas frustradas. Existe consenso de que os resultados melhoram significativamente conforme a equipe vai adquirindo experiência.

Os resultados do presente estudo evidenciam uma série de dificuldades que se apresentam ao iniciar um programa de FIV em instituição de ensino, sem fins lucrativos e voltada à população mais carente, com recursos altamente limitados e sem contar com qualquer incentivo econômico. Ainda assim, apesar do reduzido tamanho da amostra, consideramos estar no caminho certo e acreditamos que, com a prática, a nossa taxa cumulativa de gravidez tende a ser semelhante àquelas obtidas nos centros de referência, porém com custo e incidência de complicações significativamente inferiores.

\section{ABSTRACT}

Purpose: to evaluate the first in vitro fertilization results at a medical university using low-cost programmed cycles.

Methods: from May to December 2002, 66 programmed cycles of in vitro fertilization were carried out using norethisterone acetate, clomiphene citrate and human chorionic gonadotrophin ( $h C G$ ). The ovarian follicle aspiration was guided by ultrasonography, 34 to $36 \mathrm{~h}$ after the administration of $h C G$, and the embryo transfer, $48 \mathrm{~h}$ after puncturing. The diagnosis of clinical pregnancy was defined when a pulsating heart was detected by transvaginal ultrasonography.

\author{
Custo medicação** \\ por ciclo (R\$)
}

150,00

Custo medicação**

por embrião (R\$)

96,00

$\begin{array}{cc}\begin{array}{c}\text { Número de embriões } \\ \text { por tranferência }\end{array} & \begin{array}{c}\text { Custo medicação** } \\ \text { por ciclo (R\$) }\end{array} \\ 1,56 & 150,00\end{array}$

3700,00
Results: the cycle cancelation rate was $21.2 \%$. An average of 2.8 follicles and 1.7 oocytes were obtained per puncture. In $79.6 \%$ of the punctured cycles it was possible to retrieve oocytes and 69\% of them were fertilized. The number of embryos per transfer was 1.5. Some level of difficulty occurred in $10.2 \%$ of the aspiration procedures and $32.4 \%$ in the embryo transfer. The obtained pregnancy rate was 10,8\% per transfer; however, the drug cost per transferred embryo was only $R \$ 96,00$.

Conclusion: the difficulty to begin an in vitro fertilization program in a medical school, not aiming at profits and to help a population without financial possibilities, became evident. After progressive experience, the cumulative pregnancy rate tends to be similar to that of reference centers, but with significantly lower costs and incidence of complications.

KEYWORDS: In vitro fertilization. Assisted reproduction. Clomiphene. Low cost.

\section{Referências}

1. Steptoe PC, Edwards RG. Birth after the reimplantation of a human embryo. Lancet 1978; 2:366.

2. Luna F. Assisted reproductive technology in Latin America. Some ethical and sociocultural issues. In: Vayena E, Rowe PJ, Griffin PD, editors. Current practices and controversies in assisted reproduction. $1^{\text {st }}$ ed. Geneva: WHO Library; 2002. p.31-40.

3. Barbosa CP, Badra GH, Carvalho WAP, et al. Fatores de risco ambientais e ocupacionais para a saúde reprodutiva masculina. Femina 2002; 30:465-9.

4. Gabiatti JRE, Faúndes A, Bahamondes L, Faúndes D, Ramos M, Fazano F. A experiência de iniciar um programa de fertilização in vitro em Hospital Universitário. Reprod Clim 1998; 13:221-7. 
5. Lopata A, Brown JB, Leeton JF, McTalbot JM, Wood C. In vitro fertilization of preovulatory oocytes and embryo transfer in infertile patients treated with clomiphene and human chorionic gonadotropin. Fertil Steril 1978; 30:27-35.

6. Trounson AO, Leeton JF, Wood C, Webb J, Wood J. Pregnancies in humans by fertilization in vitro and embryo transfer in the controlled ovulatory cycle. Science 1981; 212:681-2.

7. Tognotti E, Bagnoli VR, Franco Júnior JG, Zugaib M. Captação de oócitos programado com estimulação ovariana fixa. Reprodução 1989; 4:42-54.

8. Templeton A, van Look P, Lumsden MA, et al. The recovery of pre-ovulatory oocytes using a fixed schedule of ovulation induction and follicle aspiration. Br J Obstet Gynaecol 1984; 91:148-54.

9. Braude PR, Bright MV, Douglas CP, et al. A regimen for obtaining mature human oocytes from donors for research into human fertilization in vitro. Fertil Steril 1984; 42:34-8.

10.Frydman R, Rainhorn JD, Forman R, et al. Programmed oocyte retrieval during routine laparoscopy and embryo cryopreservation for later transfer. Am J Obstet Gynecol 1986; 155:112-7.

11.Jones HW Jr. The time has come. Fertil Steril 1996; 65:1090-2.

12.Ueno J, Tognotti E, Grassiotto OR, Pinotti JA. Fertilização in vitro: idade limite. Reprodução 1992; 7:29-33.

13.Templeton A. Infertility and the establishment of pregnancy: overview. Br Med Bull 2000; 56:577-87.

14.Templeton A. Assessing the outcome of IVF. Ann N Y Acad Sci 2000; 900:345-50.

15. Messinis IE, Templeton A, Angell R, Aitken J. A comparison of fixed regimens for obtaining human cleaving oocytes for research purposes. Br J Obstet Gynaecol 1986; 93:39-42.
16.Hassan HA, Saleh HA, Khalil O, Baghdady I, Ismaiel I. Double oocyte aspiration may be a solution for empty follicle syndrome: case report. Fertil Steril 1998; 69:138-9.

17.Quintans CJ, Donaldson MJ, Blanco LA, Pasqualini RS. Empty follicle syndrome due to human errors: its occurrence in an in-vitro fertilization programme. Hum Reprod 1998; 13:2703-5.

18.Perin PM, Maluf M. Influência de dois catéteres de transferência de embriões sobre as taxas de gestação em um programa de fertilização in vitro humana. Reprod Clim 1999; 14:81-8.

19. Olivennes F, Frydman R. Friendly IVF: the way of the future? Hum Reprod 1998; 13:1121-4.

20.Templeton A. Avoiding multiple pregnancies in ART: replace as many embryos as you like - one at a time. Hum Reprod 2000; 15:1662-5.

21.Hazekamp J, Bergh C, Wennerholm UB, Hovatta O, Karlstrom PO, Selbing A. Avoiding multiple pregnancies in ART: consideration of new strategies. Hum Reprod 2000; 15:1217-9.

22. Cain JM. Ethical guidelines in the prevention of iatrogenic multiple pregnancy. Int J Gynaecol Obstet 2000; 71:293-4.

23.Schreiner-Engel P, Walther VN, Mindes J, Lynch L, Berkowitz RL. First-trimester multifetal pregnancy reduction: acute and persistent psychologic reactions. Am J Obstet Gynecol 1995; 172:541-7.

24.Evans MI, May M, Drugan A, Fletcher JC, Johnson MP, Sokol RJ. Selective termination: clinical experience and residual risks. Am J Obstet Gynecol 1990; 162:1568-72.

25.Zegers-Hochschild F. The Latin American registry of assisted reproduction. In: Vayena E, Rowe PJ, Griffin PD, editors. Current practices and controversies in assisted reproduction. $1^{\text {st }}$ ed. Geneva: WHO Library; 2002. p.355-62. 